\title{
Melanoma Cell Detection in LyMPH NODES HISTOPATHOLOGICAL IMAGES USING DEEP LEARNING
}

\author{
Salah Alheejawi, Richard Berendt, Naresh Jha and Mrinal Mandal \\ University of Alberta, Edmonton, Alberta, Canada
}

\begin{abstract}
Histopathological images are widely used to diagnose diseases including skin cancer. As digital histopathological images are typically of very large size, in the order of several billion pixels, automated identification of all abnormal cell nuclei and their distribution within multiple tissue sections would assist rapid comprehensive diagnostic assessment. In this paper, we propose a technique, using deep learning algorithms, to segment the cell nuclei in Hematoxylin and Eosin $(H \& E)$ stained images and detect the abnormal melanocytes within histopathological images. The Nuclear segmentation is done by using a Convolutional Neural Network (CNN) and hand-crafted features are extracted for each nucleus. The segmented nuclei are then classified into normal and abnormal nuclei using a Support Vector Machine classifier. Experimental results show that the CNN can segment the nuclei with more than $90 \%$ accuracy. The proposed technique has a low computational complexity.
\end{abstract}

\section{KEYWORDS}

Histopathological image analysis, Nuclear segmentation, Melanoma Detection, Deep learning.

\section{INTRODUCTION}

Primary cutaneous melanomas include a range of potentially lethal melanocytic neoplasms which most often present in skin as an archetypical macular growth phase lesion comprised of an in-situ component plus a papillary dermal component of similar cytomorphology. The diagnosis depends upon the histomorphological identification of abnormal melanocytes forming radial and vertical growth phase neoplastic cellular infiltrates which by invasion and widespread metastasis can secondarily involve regional lymph nodes and ultimately any other part of the body. As per recent statistics, it is estimated that about 100,350 new cases of melanoma cancer will be diagnosed in United States alone in 2020, which will result in about 6,850 deaths [1]. The early diagnosis of melanoma is very important as it helps to increase the chances of successful treatment and the survival rate. The Computer-aided diagnosis (CAD) techniques can effectively help doctors to diagnose and detect the melanoma in early stages [2]. The digitized histopathological slides, which are typically obtained by staining and scanning the biopsy slides of the skin tissue, can provide the cell morphological features with a high resolution. The digitized slides are known as Whole Slide Images (WSIs) and with help of CAD techniques that will permit the pathologist for precise diagnosis [3]. Pathologists usually use H\&E stained images, because the morphological features of the melanocytes and other cells become vividly clear. In H\&E stained image, the cell nuclei contain chromatin and that can be observed in blue shade while the cytoplasm and other connective tissues are observed with varying shades of pink.

Fig. 1 shows an H\&E stained histopathological image of metastatic melanoma within regional lymph nodes (amidst adjacent salivary gland). The Lymph node cross-sections in the image are 
Signal \& Image Processing: An International Journal (SIPIJ) Vol.11, No.4, August 2020

contoured with black color, and melanoma metastases are contoured in green color. Note that in this image the metastases are contoured manually by morphology. In the zoomed patch, it is observed that the abnormal melanocytes appear with irregularity in shape and color intensity $[4,5]$.

Several techniques have been proposed to segment the cell nuclei in histopathological images [611]. $\mathrm{Xu}$ et al. [6] proposed an automated technique (henceforth referred to as the Watershed+Voting technique) to segment the cell nuclei in H\&E stained images. The technique detects the nuclei seeds by using voting areas and segments the nuclei cells using marked watershed algorithm. The technique provides a good performance with high computational complexity due the seed detection algorithm. Xu et al. [11] also proposed cell nuclei segmentation technique (henceforth referred to as the gLoG+mRLS technique) using generalized Laplacian of Gaussian (gLoG) filters to detect the seeds nuclei and multiple Radial Lines Scanning (mRLS) algorithm to segment the cells. The mRLS uses high gradient pixel locations and shape information to accurately segment the cell nuclei.

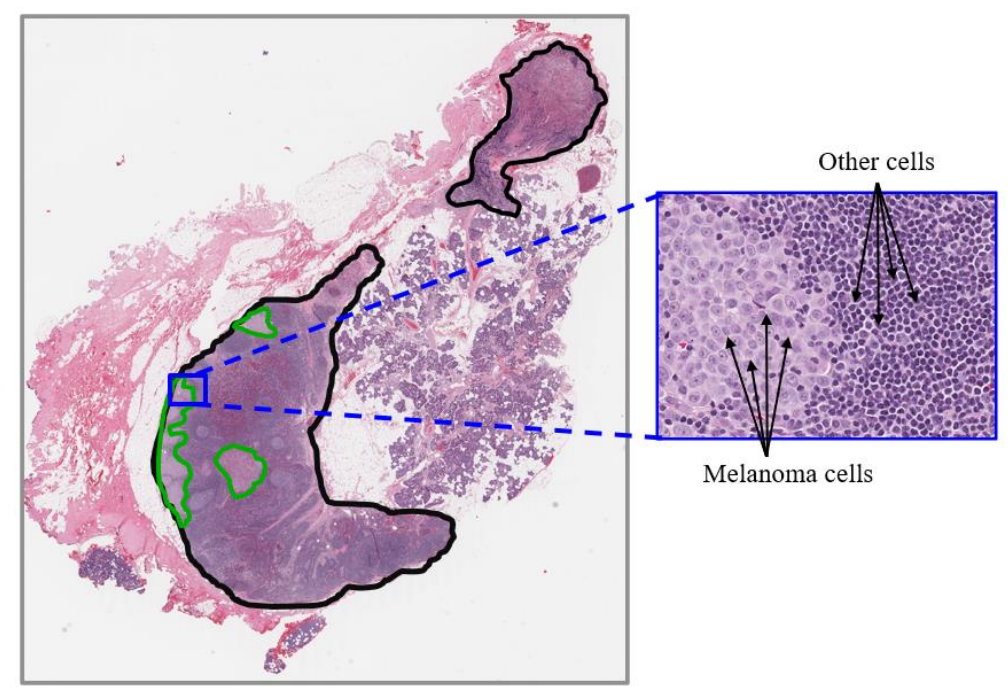

Figure 1. Example of an H\&E stained lymph node tissue image. Lymph nodal tissue is contoured in black, and metastatic melanoma deposits are contoured in green.

The techniques [6-11] mentioned above are generally based on extracted hand-crafted features that require significant time to calculate. The deep learning algorithms using CNN have been recently been used successfully in medical image analysis. The CNN models can train the feature extraction process to provide high performance with low computational complexity in many different tasks (e.g. classification, detection or segmentation [12-13]). Basrinarayanan et al. [14] proposed the SegNet architecture for object segmentation. The architecture uses a number of sampling and upsampling layers for extracting the features in hierarchical levels. Ronneberger et al. [15] proposed the U-Net architecture for biomedical image segmentation. The U-Net architecture has encoder and decoder sides with number of sampling and upsampling layers, respectively. The upsampling layer outputs are enhanced by concatenating them with features from the encoder side.

In this paper, we propose an automated technique to segment the cell nuclei and differentiate melanoma from non-melanoma cells using a Support Vector Machine (SVM) classifier. The technique uses a CNN architecture to segment the cell nuclei on H\&E stained images. The proposed CNN used several convolutional layers with different size of filters. Experimental 
Signal \& Image Processing: An International Journal (SIPIJ) Vol.11, No.4, August 2020

results demonstrate high accuracy and low computational complexity of the proposed technique compared to the state-of-the-art techniques.

The organization of the paper is as follows. Section 2 describes the dataset used to train and evaluate the proposed technique. Section 3 describes the proposed technique in detail. Section 4 presents the performance evaluation, followed by the conclusion in Section 5.

\section{DATA DESCRIPTION}

In this section, we present the details of the training and testing dataset to evaluate the performance of the proposed nuclei segmentation and cell classification technique. The digitized biopsies were collected at the Cross Cancer Institute, University of Alberta, Edmonton, Canada in accordance with the protocol for the examination of specimens with skin melanoma. Standard Neutral Buffered formalin-fixed paraffin-embedded tissue blocks of these biopsies were cut into thin slices (e.g., $4 \mu \mathrm{m}$ for light microscope). These slices were then mounted to glass slides and stained using H\&E stain [5]. The WSIs were obtained by scanning the H\&E slides using aperio scanscope scanning system under 40X magnification. The size of a WSI is typically around $40,000 \times 60,000$ pixels (in color) and each WSI contains thousands of cell nuclei. The image dataset consists of 9 WSIs of lymph node tissue.

\section{Proposed Technique}

The schematic of proposed technique is shown in Fig. 2 which consists of two modules: CNNbased nuclei segmentation and nuclei classification. The details of each module are presented in the following.

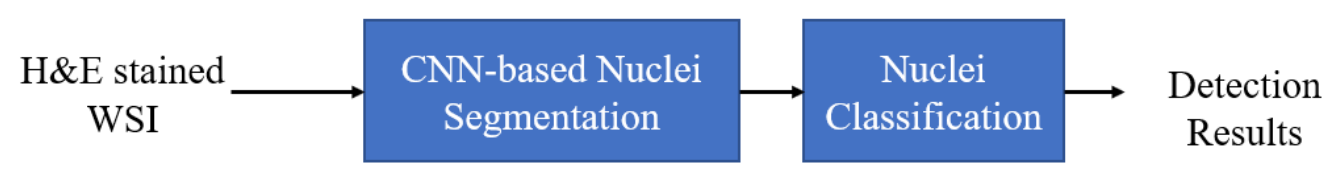

Figure 2. Schematic of the proposed melanoma detection technique.

\subsection{CNN-based Nuclei Segmentation}

In this module, the input H\&E stained images are segmented into cell nuclei and background. The nuclei segmentation is done by using the proposed CNN architecture, henceforth referred to as the NS-Net architecture (Nuclei Segmentation Net). The NS-Net architecture, shown in Fig. 3, consists of five convolutional layers (shown in gray color) and one softmax (shown in pink) followed by the pixel classification layer (shown in blue). The convolutional layer in the NSarchitecture consists of three operations: convolution, batch normalization [16], and activation [17]. A brief description of each operation is presented in the following:

(i) Convolution: Let $f_{l-1}$ denote the (3D) feature map generated in the convolutional layer $l-1$. In the convolution layer $l$, the feature map $f_{l-1}$ is convolved with a (3D) filter $F_{j}$ :

$$
R_{l, j}=f_{l-1} * F_{j}, \quad j=1,2, . ., N
$$

where $N$ is the number of filters (which is also known as the depth of the layer $l$ ), $R_{l, j}$ is the (2D) output corresponding to the $j$ th convolution filter. Note that for the first convolution layer $(l=1)$, the input image is considered as $f_{0}$. 
(ii) Batch normalization: During the CNN training, the convolution outputs $R_{l, j}$ corresponding to all images in a mini-batch (of $S$ images) are considered. In this work, we have used $S=8$. The $R_{l, j}$ is made zero mean with unit variance as follows:

$$
\hat{R}_{l, j}=\frac{R_{l, j}-\mu}{\sigma}
$$

where $\mu$ and $\sigma$ are the mean and standard deviation of all $\left\{R_{l, j}\right\}$ in a mini-batch.

The normalized $\hat{R}_{l, j}$ is scaled with $\gamma$, and a bias $\beta$ is added as follows:

$$
y_{l, j}=\gamma \hat{R}_{l, j}+\beta
$$

Note that Eqs. 2 and 3 are applied in both training and testing modes. Eq. (2) is applied with $S=8$ (the mini-batch size) and $S=1$, in training and testing mode, respectively. The $\gamma$ and $\beta$ are trainable parameters, and are updated iteratively during the backpropagation.

(iii) Activation: In this step, an activation function is applied on the batch normalized output $y_{j}$. In this work, the Rectified Linear Unit (ReLU) activation is used. The output of the ReLU activation function $f_{l, j}$ can be expressed as follows.

$$
f_{l, j}=\max \left(0, y_{l, j}\right)
$$

The overall output of convolutional layer $l$, which will be passed on to the next layer is as follows:

$$
f_{l}=\left\{f_{l, j}, j=0,1, \ldots N\right\}
$$

In this architecture, the features are extracted in hierarchical levels by using convolutional filters of different sizes. The change on the convolutional filters can precisely locate the object boundaries that need to be segmented. Most existing CNN architectures include pooling layers. In our experiment, it has been found that the pooling leads to loss of the spatial information that carries important texture and shape features of the nuclei. Therefore, the pooling layer has been omitted in the proposed architecture. Table 1 shows the number and the size of filters in each layer of the NS-Net architecture. The NS-Net architecture is trained and evaluated using a dataset of 24 high resolution H\&E stained image patches (each with $1920 \times 2500$ color pixels) obtained from the WSI dataset described in Section 2. Each image patch is divided into overlapping blocks of $64 \times 64$ color pixels to obtain 458 block-images. The total number of block-images will be 10,992 (i.e. $24 \times 458$ ) and it is divided into $70 \%$ for training, $15 \%$ for validation and $15 \%$ for testing. The entropy loss function with the stochastic gradient descent with momentum (SGDM) optimizer is used to train the NS-Net architecture [18]. Fig. 4 (a) shows an input H\&E stained image and (b) shows the masked nuclei image obtained using the NS-Net architecture.

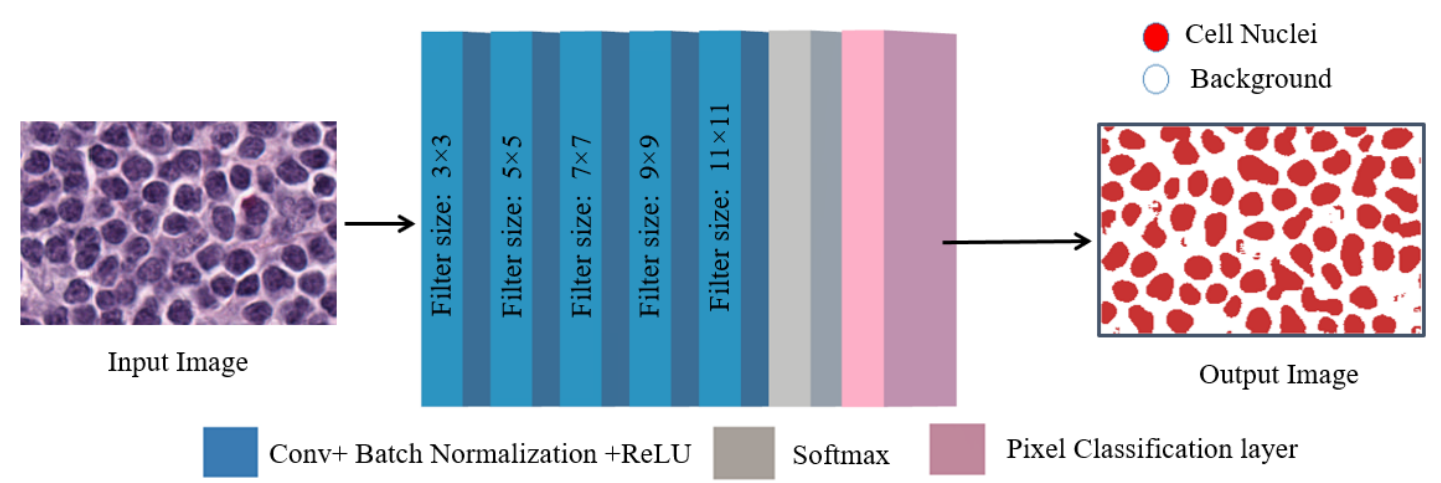

Figure 3. The proposed NS-Net architecture for nuclei segmentation. 
Signal \& Image Processing: An International Journal (SIPIJ) Vol.11, No.4, August 2020

Table 1. Details of the NS-Net architecture with 5 convolutional layers. Input image size: $\mathrm{M} \times \mathrm{N}$ pixels (color). Number of classes: $\mathrm{C}$.

\begin{tabular}{ccccc}
\hline & $\begin{array}{c}\text { Number of } \\
\text { Filters }\end{array}$ & $\begin{array}{c}\text { Number of } \\
\text { Channels }\end{array}$ & $\begin{array}{c}\text { Output Image } \\
\text { size }\end{array}$ & $\begin{array}{c}\text { Filter } \\
\text { size }\end{array}$ \\
\hline Layer-1 & 64 & 3 & $\mathrm{M} \times \mathrm{N} \times 64$ & $3 \times 3$ \\
\hline Layer-2 & 64 & 64 & $\mathrm{M} \times \mathrm{N} \times 64$ & $5 \times 5$ \\
\hline Layer-3 & 64 & 64 & $\mathrm{M} \times \mathrm{N} \times 64$ & $7 \times 7$ \\
\hline Layer-4 & 64 & 64 & $\mathrm{M} \times \mathrm{N} \times 64$ & $9 \times 9$ \\
\hline Layer-5 & $\mathrm{C}$ & 64 & $\mathrm{M} \times \mathrm{N} \times \mathrm{C}$ & $11 \times 11$ \\
\hline Softmax layer & - & $\mathrm{C}$ & $\mathrm{M} \times \mathrm{N} \times \mathrm{C}$ & \\
\hline
\end{tabular}

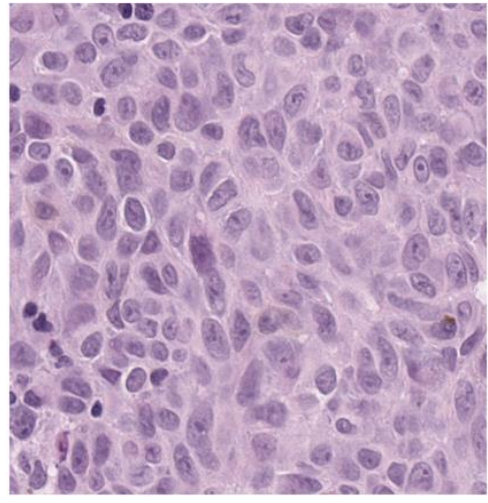

(a)

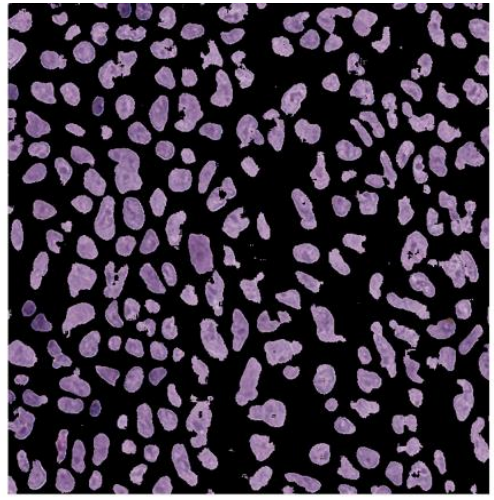

(b)

Figure 4. Segmentation results. (a) An input image patch (b) the segmented image patch obtained using the NS-Net architecture.

\subsection{Nuclei Classification}

In this module, the segmented nuclei obtained using the NS-Net architecture is classified into two classes based on hand-crafted features. The feature vector consists of 18 first-order features, 9 Histogram of Oriented Gradient features, 24 Haralick texture features and 3 Morphological features. The features are extracted for each pre-segmented cell nuclei and described briefly as follows:

(i) First-order features: it includes six histogram-based features: mean, standard deviation, third moment, smoothness, entropy, and uniformity for 3-channels (R, G and B) to obtain 18 features $(6 * 3)$.

(ii) Histogram of Oriented Gradient (HOG) features: it measures the gradient of 9 orientations in localized portions of the segmented nuclei image. A 1x9 HOG Feature Vector (HOGFVb) is computed for each non-overlapping block of 8x8 pixels (from the 64 gradient values). Each segmented cell, depending on the cell size, may contain several HOGFVs. The HOGFVb's from different blocks corresponding to a cell are summed and an overall 1x9 HOGFVn is obtained for a cell nucleus. Fig. 5 shows an example of HOG feature extraction of a cell nucleus. Note that melanoma cells having larger size (compared to other cells) typically contain more HOGFVb's than other cells, and this can result in a large magnitude of HOGFVn's. Also, the HOGFVn's corresponding to the melanoma cells tend to have non-uniform distribution compared to other cells which typically have uniform distribution.

(iii) Haralick texture features: it calculated from a Gray Level Co-occurrence Matrix, (GLCM). It includes GLCM features such as the correlation, energy, homogeneity, 
Signal \& Image Processing: An International Journal (SIPIJ) Vol.11, No.4, August 2020

contrast, entropy, and dissimilarity in 4 directions (i.e., $0^{\circ}, 45^{\circ}, 90^{\circ}$ and $135^{\circ}$ ) to obtain 24 features $(6 * 4)$.

(iv) Morphological features: it includes the eccentricity, solidity, and the ratio of major and minor axes of the cell nuclei to obtain (3) features.

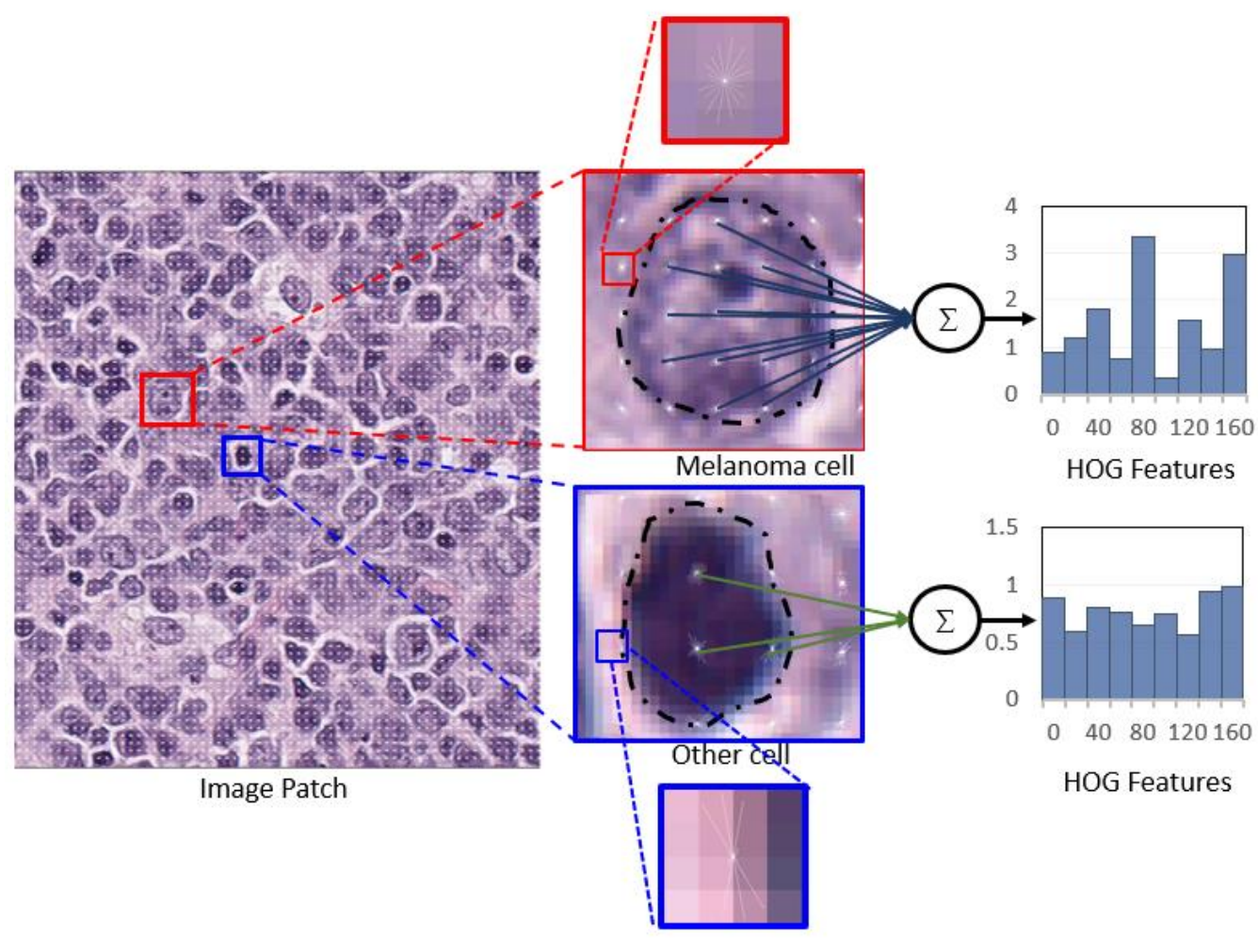

(a)

(b)

(c)

Figure 5. Example of HOG feature extraction for a cell nucleus. (a) An image patch with segmented cell nuclei and overlapped gradient orientation. (b) Blown-up of image of two nuclei (melanoma and other cells). (c) The 1x9 HOGFVn's of the cell nuclei.

The extracted feature vectors (with dimension $1 \times 54$ ) of each cell nuclei are then classified into normal and melanoma using an SVM classifier [19-20]. The SVM is very efficient supervised classifier that can handle even a non-linearly separable features and create hyperplane to separate abnormal features from the normal ones. In the proposed technique, the SVM model is trained and tested on 1,388 cell nuclei ( $70 \%$ for training and $30 \%$ for testing) obtained from $9 \mathrm{H} \& \mathrm{E}$ stained WSI of lymph nodes.

\section{RESUltS AND DisCUSSIONS}

In this section, we present the performance of the proposed nuclei segmentation and classification technique. The performance of the segmentation technique is presented first followed by the performance of the classification technique.

\subsection{Segmentation performance}

The details of the NS-Net training were presented in Section 3.1. The segmentation performance is evaluated using 1,649 $\mathrm{H} \& \mathrm{E}$ stained lymph node block images (each with $64 \times 64$ color pixels). 
Signal \& Image Processing: An International Journal (SIPIJ) Vol.11, No.4, August 2020

The segmentation performance of the proposed technique is evaluated and compared with handcrafted feature-based algorithms: gLoG+mRLS and Voting+Watershed techniques as well as CNN-based techniques using SegNet and U-Net architectures. The segmentation performance is evaluated using Precision, Accuracy and BF-score [21] measures defined as follows:

$$
\begin{gathered}
\text { Precision }=\frac{T P}{T P+F P} \times 100 \% \\
\text { Accuracy }=\frac{T P+T N}{T P+F P+F N+T N} \times 100 \% \\
B F \text { Score }=\frac{2 T P * T N}{T P * T N+2 T N * F P+T N * F N} \times 100 \%
\end{gathered}
$$

where $T P, T N, F N$ and $F P$ denote the number of true positives, true negatives, false negatives and false positives, respectively. Table 2 shows the segmentation performance of different techniques. It is observed that the deep learning algorithms provide excellent performance compared to the classical feature-based algorithms. This is because the classical features are less sensitive to the diversity of the cell nuclei in the skin tissue. For example, the melanoma cells tend to have light and inhomogeneous color (see Fig. 1) and that causes miss detection of the melanoma cells in the gLoG+mRLS and Voting+Watershed techniques.

Table 2. Segmentation Performance of the deep learning algorithms and the classical feature-based algorithms.

\begin{tabular}{|l|l|l|l|l|}
\hline Technique: & Precision & Accuracy & BF-Score & $\begin{array}{l}\text { Execution } \\
\text { time (in s) }\end{array}$ \\
\hline Voting+Watershed [6] & 78.24 & 83.64 & 81.31 & 143.71 \\
\hline gLoG+mRLS [11] & 79.27 & 76.67 & 68.46 & 128.57 \\
\hline SegNet [14] & 84.16 & 87.84 & 85.81 & 15.37 \\
\hline U-Net [15] & 87.41 & 78.79 & 69.63 & 20.82 \\
\hline NS-Net & 87.20 & 90.21 & 88.52 & 14.27 \\
\hline
\end{tabular}

In this work, the NS-Net, SegNet and U-Net architectures are trained with the same number of training images. The NS-Net architecture is also evaluated with CNN architecture in terms of the required parameters need to be train as shown in Table 3 .

Figs. 6 (b)-(f) show the subjective segmentation performance of Voting+Watershed [6], gLoG+mRLS [11], SegNet [14], U-Net [15] and the proposed NS-Net architecture, respectively. It is observed that the NS-Net architecture provides excellent nuclei segmentation, whereas gLoG+mRLS, Voting+Watershed techniques miss a few cell nuclei due to the inhomogeneity in the cell nuclei color. It is also observed that the U-Net architecture does not perform well compared to the other techniques because the overfitting due the large number of the filters that are used in the cell nuclei segmentation.

Table 3. Properties of CNN architectures used in performance evaluation.

\begin{tabular}{|c|c|c|c|c|}
\hline $\begin{array}{c}\text { CNN } \\
\text { Architecture }\end{array}$ & $\begin{array}{c}\text { Convolutional } \\
\text { layers }\end{array}$ & $\begin{array}{c}\text { No. of Trained } \\
\text { parameters }\end{array}$ & Filter size & No. of Filters \\
\hline SegNet [14] & 8 & 225,542 & $3 \times 3$ & 64 \\
\hline U-Net [15] & 11 & 905,472 & $3 \times 3$ & $(64,128,256)$ \\
\hline NS-Net & 5 & 150,336 & $(3 \times 3)-(11 \times 11)$ & 64 \\
\hline
\end{tabular}




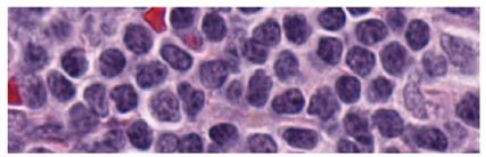

(a) An H\&E stained image patch

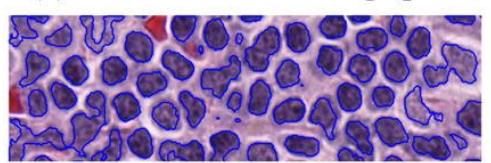

(d) SegNet [14]

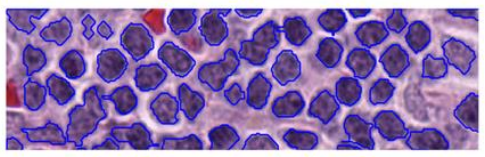

(b) Voting+Watershed [6]

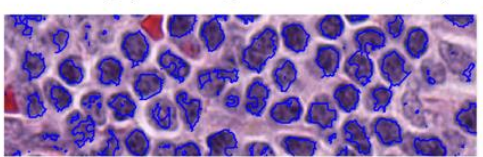

(e) U-Net [15]

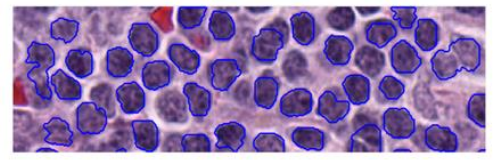

(c) gLoG+mRLS [11]

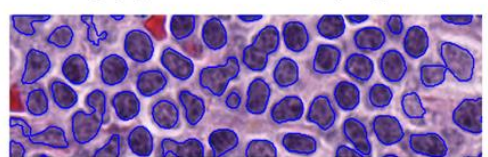

(f) NS-Net

Figure 6. Subjective comparison of cell nuclei segmentation results (contoured in blue color) (a) original test image, (b)-(f) Segmentation results for Voting+Watershed [6], gLoG+mRLS [11], SegNet [14], U-Net [15] and NS-Net techniques, respectively.

\subsection{Classification performance}

The classification performance is evaluated using $240 \mathrm{H} \& \mathrm{E}$ stained lymph node nuclei. As explained in Section 3.2, a $1 \times 54$ size feature vector is obtained for each nucleus. The obtained $1 \times 54$ feature vectors include 18 first-order features, 9 Histogram of Oriented Gradient features, 24 Haralick texture features and 3 Morphological features. A nucleus is classified based on its feature vector using the SVM model.

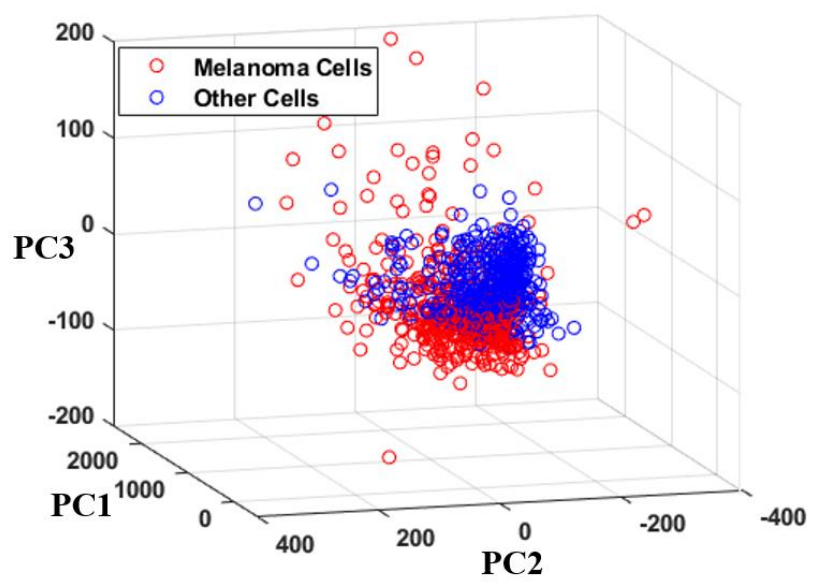

Figure 7. Three principal components (PC-1, PC-2, and PC-3) of the $1 \times 54$ features.

To demonstrate the discrimination ability of the features, the obtained features are analysed using Principal Component Analysis (PCA) to capture the data and features variations. The PCA technique is applied on the training cells nuclei (972 cell nuclei: 486 Melanoma and 486 other nuclei). Fig. 7 visualizes the feature variance of melanoma and other cells with respect to three principal components that are extracted from the nuclei features. Analyzing the feature vectors and the principal components, it has been found that the first principal component (PC1) has high positive association with some of the first order and Haralick features, whereas the second and third principal components have positive association with some of the histogram and morphological features. In this experiment, the first, second and third principal components capture $82 \%$ of feature variance. The remaining (51) dimensions add $18 \%$ of feature variance. In this work, all 54 features are used for the SVM classification. 
Signal \& Image Processing: An International Journal (SIPIJ) Vol.11, No.4, August 2020

The classification performance is evaluated in terms of the Precision, Recall and Accuracy defined in Section 4.1. The results are shown in Table 4. The SVM classifier has been evaluated with linear, polynomial and Gaussian kernels [18]. It is observed that the SVM classifier with Gaussian kernel provides a superior performance over the other two kernels. Fig. 8 shows the nuclei classification results obtained using the Gaussian kernel, where the melanoma and nonmelanoma nuclei are contoured in red and blue colors, respectively.

Table 4. Performance of the nuclei classification using different SVM kernels.

\begin{tabular}{|c|c|c|c|}
\hline \multirow{2}{*}{$\begin{array}{c}\text { Evaluation } \\
\text { Measures }\end{array}$} & \multicolumn{3}{|c|}{ SVM Kernel } \\
\cline { 2 - 4 } & Linear & Polynomial & Gaussian \\
\hline Precision & 77.43 & 57.28 & 80.04 \\
\hline Recall & 93.14 & 96.65 & 97.42 \\
\hline Accuracy & 80.52 & 57.28 & 85.72 \\
\hline
\end{tabular}

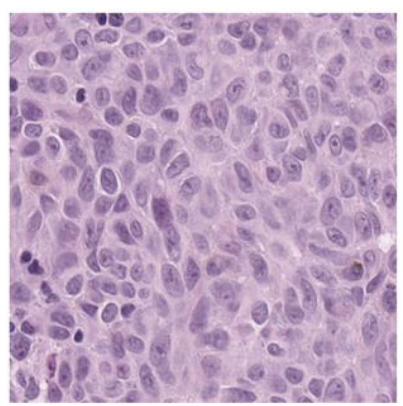

(a)

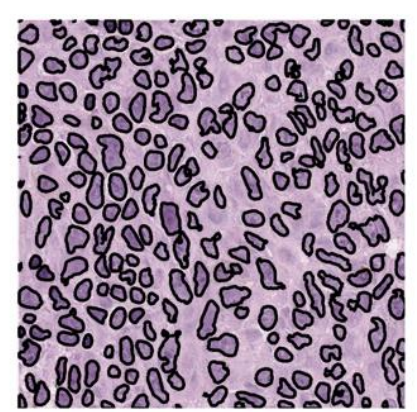

(b)

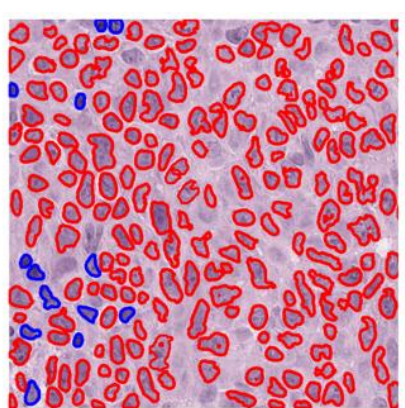

(c)

Figure 8. Example of classification results. (a) NS-Net input image (b) NS-Net segmented output image (c) Classified image obtained using SVM, where melanocytes and other cell nuclei are contoured with red and blue color, respectively.

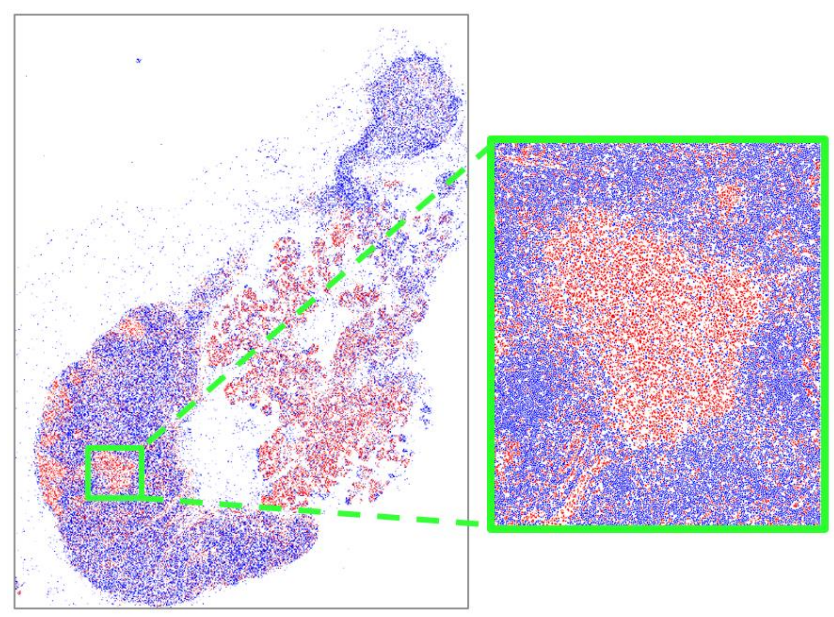

Figure 9. Visual example of nuclei segmentation and classification obtained by the proposed technique). The classified melanoma cells are shown in red color, whereas the non-melanoma cells are shown in blue color.

Fig. 9 shows the segmentation and classification results of the proposed technique for the lymph nodes in Fig.1. It is observed that the melanoma cells (in red color) are dense on the melanoma metastasises, whereas the other cells are dense on the normal tissue of the lymph nodes. The 
Signal \& Image Processing: An International Journal (SIPIJ) Vol.11, No.4, August 2020

dense areas of the melanoma cells can be used to mask the melanoma regions on the lymph node tissue. It is also noticed that there are some areas out of the lymph node tissue contains melanoma cells and that can be processed by applying an initial lymph node segmentation technique [23].

\section{CONClusions}

This paper proposes an automated technique to detect melanoma nuclei in lymph node histopathological images. The technique segments the cell nuclei in H\&E stained image using a deep learning NS-Net architecture. The NS-Net architecture segments the image into background and cell nuclei regions. The segmented nuclei are then classified into melanoma and other cell nuclei using an SVM classifier with Gaussian kernel. The proposed CNN architecture provides an excellent segmentation performance with a low computational complexity. The future work includes identifying the region of interest (ROI) with clusters of melanoma nuclei and derive prognostic information such as proliferation index.

\section{REFERENCES}

[1] Rebecca L. Siegel, Kimberly D. Miller \& Ahmedin Jemal, (2020), "Cancer Statistics, 2020," CA: A Cancer Journal for Clinicians, vol. 70, No. 1, pp.7-30.

[2] Brochez, Lieve, Verhaeghe, Evelien, Grosshans, Edouard, et al, (2002), "Inter-observer variation in the histopathological diagnosis of clinically suspicious pigmented skin lesions". J. Pathol. 196(4), pp.459-466.

[3] Cheng Lu, Muhammad Mahmood, Naresh Jha \& Mrinal Mandal, (2013), "Automated segmentation of the melanocytes in skin histopathological images". IEEE J. Biomed. Health Inf. 17(2), pp. 284296.

[4] Marcial G. Rojo, Ana M. Castro, \& Luis Gonçalves, (2011), "COST action "eurotelepath": digital pathology integration in electronic health record, including primary care centres," Diagnostic Pathology 6, S6 (2011), pp. S1-6.

[5] Ronald S. Weinstein, Anna R. Graham, Lynne C. Richter, et al. (2009), "Overview of telepathology, virtual microscopy, and whole slide imaging: prospects for the future," Human Pathology, vol. 40, no. 8, pp. 1057-1069.

[6] Hongming Xu, Cheng Lu \& Mrinal Mandal (2013), “An efficient technique for nuclei segmentation based on ellipse descriptor analysis and improved seed detection algorithm". IEEE J. Biomed. Health Inf. 18(5), pp. 1729-1741.

[7] Olcay Sertel, (2012), "Image analysis for computer-aided histopathology," Ph.D. dissertation, The Ohio State University.

[8] Juliana M Haggerty, Xiao N Wang, Anne Dickinson, Chris J O’Malley \& Elaine B Martin, (2014), "Segmentation of epidermal tissue with histopathological damage in images of haematoxylin and eosin stained human skin". BMC Med. Imaging. 14(1), pp. 7.

[9] Jierong Cheng, Jagath C. Rajapakse, (2009), "Segmentation of clustered nuclei with shape markers and marking function," IEEE Transactions on Biomedical Engineering, vol. 56, no. 3, pp. 741-748.

[10] Bahram Parvin, Qing Yang, Ju Han, Hang Chang, Bjorn Rydberg, Mary Helen \& Barcellos-Hoff, (2007), "Iterative voting for inference of structural saliency and characterization of subcellular events," IEEE Transactions on Image Processing, vol. 16, no. 3, pp. 615-623.

[11] Hongming Xu, Cheng Lu, Richard Berendt, Naresh Jha \& Mrinal Mandal, (2017), "Automatic nuclei detection based on generalized Laplacian of Gaussian filters," IEEE Journal of Biomedical and Health Informatics (JBHI), vol.21, no.3, pp.826-837.

[12] Jonathan Long, Evan Shelhamer \& Trevor Darrell, (2015), "Fully convolutional networks for semantic segmentation," in CVPR, pp. 3431-3440.

[13] David Eigen, \& Rob Fergus, (2015) "Predicting depth, surface normals and semantic labels with a common multi-scale convolutional architecture", in Proceedings of the International Conference on Computer Vision, pp. 2650-2658.

[14] Vijay Badrinarayanan, Alex Kendall, \& Roberto Cipolla (2015). "SegNet: A Deep Convolutional Encoder-Decoder Architecture for Image Segmentation." arXiv. Preprint arXiv: 1511.0051. 
Signal \& Image Processing: An International Journal (SIPIJ) Vol.11, No.4, August 2020

[15] Olaf Ronneberger, Philipp Fischer, \& Thomas Brox (2015). "U-Net: Convolutional Networks for Biomedical Image Segmentation." Medical Image Computing and Computer-Assisted Intervention (MICCAI). Vol. 9351, pp. 234-241.

[16] Sergey Ioffe, \& Christian Szegedy, (2015), "Batch normalization: accelerating deep network training by reducing internal covariate shift," arXiv preprint arXiv:1502.03167.

[17] Vinod Nair, \& Geoffrey E. Hinton (2010), "Rectified linear units improve restricted Boltzmann machines," in Proceedings of the 27th international conference on machine learning, pp. 807-814.

[18] Christian Robert, (2014) "Machine learning, a probabilistic perspective," CHANCE, vol. 27, no. 2, pp. 62-63.

[19] Corinna Cortes \& Vladimir Vapnik, (1995), "Support-vector networks". Machine Learning. 20 (3), pp. 273-297.

[20]. Asa Ben-Hur, David Horn, Hava T. Siegelmann, \& Vladimir N. Vapnik, (2001) "Support vector clustering," Journal of Machine Learning Research, vol. 2, pp. 125-137.

[21] Gabriela Csurka, Diane Larlus, \& Florent Perronnin (2013), "What is a good evaluation measure for semantic segmentation?" Proc. of the British Machine Vision Conference, pp. 32.1-32.11.

[22] Hervé Abdi \& Lynne J. Williams (2010), "Principal component analysis". Wiley Interdisciplinary Reviews: Computational Statistics. 2 (4): 433-459.

[23] Salah Alheejawi, Hongming Xu \& Mrinal Mandal (2018), "Novel lymph node segmentation and proliferation index measurement for skin melanoma biopsy images" Computerized Medical Imaging and Graphics CMIG. 